\title{
TOXICITY OF INDIVIDUAL PHARMACEUTICALS AND THEIR MIXTURES TO ALIIVIBRIO FISCHERI: EVIDENCE OF TOXICOLOGICAL INTERACTIONS IN BINARY COMBINATIONS
}

\author{
Valeria Di Nica,* Sara Villa, and Antonio Finizio \\ Department of Earth and Environmental Sciences, University of Milano Bicocca, Milano, Italy \\ (Submitted 24 May 2016; Returned for Revision 13 October 2016; Accepted 13 November 2016)
}

\begin{abstract}
The combined toxicities of binary mixtures of veterinary pharmaceutical active compounds were examined using the bioluminescent bacterium Aliivibrio fischeri as a test organism (Microtox ${ }^{\circledR}$ test). Mixtures were prepared at an equitoxic ratio that corresponded to the inhibitory concentration, 10\% (IC10) of individual pharmaceutical active compounds. In addition, the toxicity was determined of a multicomponent mixture that contained all of the investigated pharmaceutical active compounds mixed at a ratio corresponding to their individual predicted no-effect concentration (PNEC) values. The experimental results were successively compared with those obtained by applying the 2 most widely used models for predicting mixture toxicity, the concentration addition (CA) and independent action (IA) models. Although the toxicity of the multicomponent mixture tested was well predicted by the CA and IA models, deviations from the model predictions were found for almost all of the binary mixtures. The deviations from the CA and IA models were greater at lower concentrations, particularly when diclofenac sodium and amoxicillin were present in the mixture. Based on these results, another hypothesis was tested, that of toxicological interactions occurring in binary mixtures (in the direction of synergistic or antagonistic effects), by applying the combination index method, which allowed for computerized quantification of synergism, the additive effect and antagonism. The application of this method confirmed, for at least half of the binary combinations, the clear presence of synergistic deviations at the lowest tested concentrations, with a tendency toward antagonism at the higher ones. In 1 case, a relevant antagonistic interaction was observed. Environ Toxicol Chem 2017;36:815-822. (C) 2016 SETAC
\end{abstract}

Keywords: Alivibrio fischeri Mixture toxicity Veterinary pharmaceuticals Combination index Synergism

\section{INTRODUCTION}

Organisms are typically exposed to a cocktail of chemicals rather than 1 individual substance. This is particularly true in surface water systems, where monitoring studies have generally detected a multitude of heterogeneous and potentially toxic substances [1-4]. Hence, the risk posed by the presence of mixtures in environmental compartments should be evaluated. During the last decade, the European Commission became aware of the problem of chemical mixtures in the environment [5] and defined the challenges that require scientific support in its communication on the combination effects of chemicals [6]. However, presently, environmental risk assessment procedures are generally developed for single substances, which may lead to a severe underestimation of the real risk facing the environment $[7,8]$.

Our understanding of the ecotoxicology of mixtures is in the first stages, because of the complexity of the variables that need to be taken into consideration [9]. The experimental assessment of the ecotoxicity of every potentially occurring mixture is not feasible and is still limited because of the wide temporal and spatial variability of compounds in a given area. In addition, obtaining a full prediction of mixture effects by using models is not easy. Two simple component-based predictive models for assessing the responses of mixtures are commonly used: the concentration addition (CA) or dose addition [10] and independent action (IA) [11] models. The CA approach is thought to be applicable to mixtures of chemicals that share a similar or common mode of action. Conversely IA, which is also known as response addition,

\footnotetext{
This article includes online-only Supplemental Data.

* Address correspondence to v.dinica@campus.unimib.it

Published online 16 November 2016 in Wiley Online Library (wileyonlinelibrary.com).

DOI: $10.1002 /$ etc.3686
}

is widely held to be appropriate for mixtures of agents that show a dissimilar mode of action. These concepts are both based on knowledge of the concentration-response relationships of the single components. In many cases mixtures in which components had similar or dissimilar modes of action were correctly predicted with the concepts of CA and IA, respectively [12-14]. The results from the IA model usually lead to slightly lower responses than those predicted by CA, and thus CA is often used as the acceptable worst case. However, in some cases, the so-called prediction window $[15,16]$ is very small, which indicates that both models are able to predict the response of mixtures independently based on the mode of action of single components $[7,17,18]$.

By definition, the CA and IA concepts ignore synergistic and antagonistic effects, and both models assume that the components of a mixture do not interact chemically or in toxicokinetic/ toxicodynamic phases [9]. For the quantification of both phenomena, Chou and Talalay introduced the combination index method [19]. This approach was historically applied in pharmacology to understand the nature of the interactions among pharmaceutical active compounds [20], and it was more recently applied in the environmental field to study the interactions among chemical contaminants present in environmental mixtures [21-23]. In the first part of our study, the toxicity of 10 different pharmaceutical active compounds (for both veterinary and human use) to Alivivibrio fischeri (formerly Vibrio fischeri or Photobacterium phosphoreum) was determined using the Microtox ${ }^{\circledR}$ test system [24]. In this second part, we focused on pharmaceutical active compounds registered for veterinary use. In particular, we investigated the effects of a series of their binary or multicomponent mixtures on A. fischeri. In the previous study [24], we demonstrated that almost all the selected pharmaceutical active compounds act as polar-narcotic compounds toward A. fischeri. The only exception was the antibiotic chlortetracycline, which had a specific mode of action. Based on these findings, the mixture 
responses containing narcotic-type pharmaceutical active compounds should be well predicted by the CA model. In contrast, the IA approach should be more suitable for the prediction of mixture toxicities containing chlortetracycline. The binary mixtures were prepared at an equitoxic ratio that corresponded to the individual inhibitory concentration, $10 \%$ (IC10) obtained in our previous work [24]. The multicomponent mixture was prepared at a ratio corresponding to the individual predicted no-effect concentration (PNEC) value. The experimental results were compared with those obtained by the application of the CA and IA models, to assess the additive behavior of chemicals in a mixture or the existence of deviations from conceptual expectations. Finally, the nature of the pharmaceutical active compound interactions was also investigated by applying the combination index method to verify the existence of possible synergistic and/or antagonistic effects.

\section{MATERIALS AND METHODS}

\section{Chemicals and testing procedures}

The tested veterinary pharmaceutical active compound compounds were the same as those reported by $\mathrm{Di}$ Nica et al. [24]: amoxicillin, chlortetracycline, sulfamethizole, diclofenac sodium, and acetylsalycilic acid. The CAS numbers, main physicochemical properties, and individual acute toxicity data (IC50 and IC10) for A. fischeri have been reported by Di Nica et al. [24]. The stock solutions were prepared using the method described in Vighi et al. [25]. Acute toxicity (15 min) to the bioluminescent bacterium A. fischeri (purchased from Ecotox) was measured using the Microtox model 500 analyzer (Ecotox). The testing procedure was described by Di Nica et al. [24]. For binary mixtures, the tested concentrations (from 9 to 22 experimental concentrations) ranged from 0.02 to 35 toxic units, where the toxic units of the mixture correspond to the sum of the ratio between the actual concentration of the chemicals present in the mixture and their relative IC10. Solutions were buffered with $0.1 \mathrm{M}$ sodium phosphate and $\mathrm{pH}$ was adjusted to a range of 6 to 8.50 (DIN EN ISO 11358-3) using a pH meter model 250 (Denver Instruments). All tests are referred to nominal (= initial) concentrations of pharmaceutical active compounds. Tests were performed in 4 replicates. Stock solutions of sulfamethizole, amoxicillin, and acetylsalycilic acid were prepared by using dimethyl sulfoxide (DMSO). For mixtures containing these pharmaceutical active compounds, the final test samples contained a concentration of DMSO that was always below $1.5 \%(\mathrm{v} / \mathrm{v})$. As reported in our previous study [24], at this level of concentration, the influence of DMSO for low-water-solubility compounds is negligible.

\section{Experimental design for selected mixtures}

Eleven mixtures were prepared by mixing individual chemicals at equitoxic concentrations in the saline solution $(2 \% \mathrm{NaCl})$ that was used for the toxicity test. This concentration ratio was kept constant, and, using a serial dilution, different concentrations of stock solutions were tested (fixed ratio design).

The tested mixtures were as follows. First, 10 combinations of binary mixtures of compounds mixed in a ratio corresponding to their individual IC10 value (acetylsalycilic acid-amoxicillin, acetylsalycilic acid-chlortetracycline, acetylsalycilic aciddiclofenac sodium, acetylsalycilic acid-sulfamethizole, chlortetracycline-amoxicillin, chlortetracycline-diclofenac sodium, chlortetracycline-sulfamethizole, diclofenac sodiumamoxicillin, diclofenac sodium-sulfamethizole, sulfamethizoleamoxicillin, with individual IC10 values of the selected pharmaceutical active compounds shown in the Supplemental Data, Table S1). Second, 1 multicomponent mixture of the 5 compounds mixed in a ratio corresponding to their individual PNEC value (mix-PNEC: chlortetracycline-diclofenac sodiumacetylsalycilic acid-sulfamethizole-amoxicillin).

In line with the current guidelines on environmental risk assessment of pharmaceuticals [26,27], PNECs were extrapolated by applying an appropriate assessment factor to the lowest relevant observed value within the toxicity dataset available in the literature. The calculated PNEC values are reported in the Supplemental Data, Table S2.

\section{Concentration-response curve fitting}

To quantitatively describe the concentration-response curves, the observed concentration-effect data were fitted to the nonlinear regression Weibull model (Equation 1):

$$
I=(c, \beta)=1-\exp \left\{-\exp \left\{\ln \left(\beta_{2}\left(\ln (c)-\ln \left(\beta_{1}\right)\right)\right\}\right\}\right.
$$

where $I$ is the fractional response $(0 \leq \mathrm{I} \leq 1)$ in terms of the inhibition of luminescence, $c$ is the chemicals concentration and $\beta_{1}$ and $\beta_{2}$ are the parameters of the model. The $I_{x}\left(100 \times I_{x} \%\right)$ and the confidence intervals were obtained using the $R^{\circledR}$ software package [28,29].

\section{Prediction of mixture toxicities by CA and IA models}

Based on the fixed ratio of the mixture components, it was possible to express the concentration of single chemicals as a fraction of the total concentration. Therefore, it was possible to apply the CA model using Equation 2 [15]:

$$
E C x_{\text {mix }}=\left(\sum_{i=1}^{n} \frac{p_{i}}{E C_{x_{i}}}\right)^{-1}
$$

where $E C x_{\text {mix }}$ is the total concentration of the mixture that produces an $x \%$ of the effect, $p_{i}\left(C_{i} / C_{\text {mix }}\right)$ is the fraction of the component $i$ in the mixture, and $E C_{x, i}$ is the concentration of the $i^{t h}$ component when present individually and elicits the same effect $(x \%)$ as the mixture. It was possible to derive the predicted concentration-response curve using Equation 2.

The alternative IA model is commonly used to predict the mixture toxicity of substances that have different or dissimilar mechanisms of action. It may be applied using Equation 3:

$$
\begin{aligned}
E\left(C_{\text {mix }}\right) & =1-\Pi_{1=1}^{n}\left(1-E\left(C_{i}\right)\right) \text { with } C_{\text {mix }} \\
& =c_{l} . . \ldots \ldots+c_{n}
\end{aligned}
$$

where $C_{i}$ denotes the concentrations of the $i^{\text {th }}$ mixture component, $E\left(c_{i}\right)$ is its corresponding effect, and $E\left(C_{\text {mix }}\right)$ is the overall effect as a result of the mixture $[11,15]$.

\section{Application of the combination index-isobologram equation}

To highlight possible synergistic or antagonistic effects, a further analysis of the response of $A$. fischeri to pharmaceutical active compound mixtures was carried out using the combination index approach [20,30,31] (Equation 4):

$$
{ }^{n}(C I)_{x}=\sum_{j=1}^{n} \frac{(D)_{j}}{\left(D_{x}\right)_{j}}=\sum_{j=1}^{n} \frac{\left(D_{x}\right)_{1-n}\left\{\frac{[D]_{j}}{\sum_{1}^{n}[D]}\right\}}{\left(D_{m}\right)_{j}\left\{\frac{\left(f a_{x}\right)_{j}}{\left[1-\left(f a_{x}\right)_{j}\right]}\right\}^{1 / m_{j}}}
$$

where ${ }^{n}(C I)_{x}$ is the combination index for $n$ chemicals at $x \%$ inhibition, $\left(D_{x}\right)_{1-n}$ is the sum of the dose of $n$ chemicals that 
exerts $x \%$ inhibition in combination, $\left\{\left([D]_{j}>\sum[D]\right\}\right.$ is the proportionality of the dose of each of $n$ drugs that exerts $x \%$ inhibition in combination, and $\left(D_{m}\right)_{j}\left\{\left(f_{a x}\right)_{j} /\left[1-\left(f_{a x}\right)_{j}\right]\right\}^{1 / m j}$ is the dose of each drug alone that exerts $x \%$ inhibition. $D_{\mathrm{m}}$ is the median-effect dose, $f_{a x}$ is the fractional inhibition at $x \%$ inhibition, and $m$ is the slope of the median-effect plot.

From Equation 4, combination index $<1$, combination index $=1$, and combination index $>1$ indicate synergism, the additive effect, and antagonism, respectively.

The computer program CompuSyn was used to calculate the combination index values for the different mixtures at different ranges of effect levels $(10 \%, 25 \%, 50 \%, 75 \%$, and $90 \%$ of inhibition of bioluminescence) [32].

\section{RESULTS AND DISCUSSION}

\section{Experimental mixture toxicities}

Binary mixtures of pharmaceutical active compounds. Values of IC10 and IC50 were derived from the concentrationresponse curves of the tested binary pharmaceutical active compound combinations (Table 1). As shown in Table 1, the IC50 values obtained spanned over 2 orders of magnitude.

In particular, the binary mixture chlortetracycline-diclofenac sodium showed the highest toxicity $\left(\mathrm{IC} 10=0.68 \mathrm{mg} \mathrm{L}^{-1}\right.$ and IC50 $=10.1 \mathrm{mg} \mathrm{L}^{-1}$ ), followed by the diclofenac sodiumacetylsalycilic acid combination (IC10 $=18.2 \mathrm{mg} \mathrm{L}^{-1}$ and IC50 $\left.=111.6 \mathrm{mg} \mathrm{L}^{-1}\right)$. In contrast, the binary combinations containing amoxicillin showed the lowest IC50.

These results were not surprising considering that, in our previous study [24], chlortetracycline and diclofenac sodium tested singly were the most toxic pharmaceutical active compounds to $A$. fischeri $\left(\mathrm{IC} 10=2.0 \mathrm{mg} \mathrm{L}^{-1}\right.$ and $\mathrm{IC} 50=12.1$ $\mathrm{mgL}^{-1}$ for chlortetracycline; IC10 $=5.9 \mathrm{mgL}^{-1}$ and IC $50=15.9 \mathrm{mg} \mathrm{L}^{-1}$ for diclofenac sodium) whereas the amoxicillin was the least toxic $1\left(\mathrm{IC} 50=>1702 \mathrm{mg} \mathrm{L}^{-1}\right)$.

For the majority of the tested binary mixtures, a complete concentration-response relationship was obtained (Figure 1). In 4 cases the maximum percentage of inhibition reached was near $50 \%$ (acetylsalycilic acid-amoxicillin and sulfamethizoleamoxicillin 43\%; acetylsalycilic acid-sulfamethizole and chlortetracycline-amoxicillin 48\%).

Multicomponent mixtures of pharmaceutical active compounds. A complete concentration-response curve was obtained for the tested multicomponent mixture of veterinary

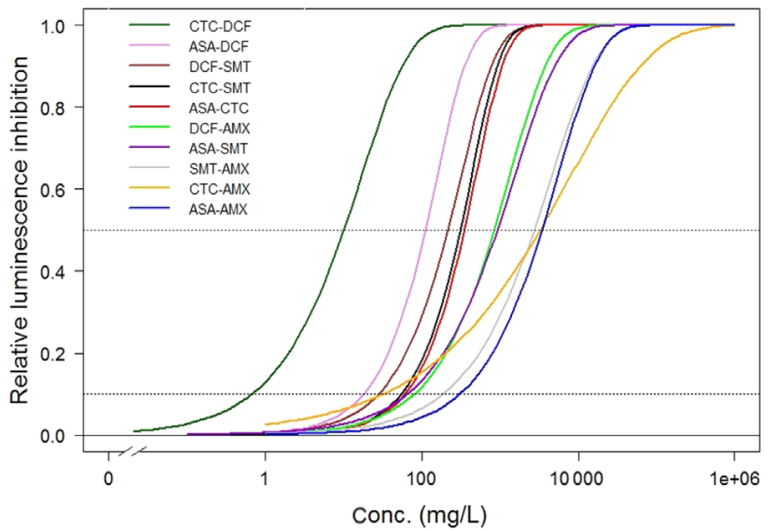

Figure 1. Concentration response curves (Weibull model) of the tested binary mixtures of veterinary pharmaceutical active compounds (dashed lines = intersections at values of individual acute toxicity data [IC50 and IC10, respectively]). $\mathrm{AMX}=$ amoxicillin; $\mathrm{ASA}=$ acetylsalycilic acid; $\mathrm{CTC}=$ chlortetracycline; $\mathrm{DCF}=$ diclofenac sodium; SMT $=$ sulfamethizole.

pharmaceutical active compounds (mixed at their PNEC ratio). The resulting IC10 and IC50 values were $25.0 \mathrm{mg} \mathrm{L}^{-1}$ and $220.7 \mathrm{mg} \mathrm{L}^{-1}$, respectively. The results obtained showed that at concentrations corresponding to the sum of individual PNECs $\left(0.16 \mathrm{mg} \mathrm{L}^{-1}\right)$, a very negligible effect was measured on $A$. fischeri; in addition, the IC10 was 2 orders of magnitude greater than the sum of the single-compound safety threshold for aquatic organisms, and at least 4 orders of magnitude above their joint measured environmental concentrations in European water bodies that are in the range of $\mathrm{ng} \mathrm{L}^{-1}$ [24].

These results are not surprising considering that PNECs have been calculated by applying an assessment factor to acute or chronic toxicity data for the most sensitive species and that $A$. fischeri is not the most sensitive for all the tested compounds, as demonstrated in our previous study [24].

Predicted mixture toxicity by $C A$ and IA models and comparison with experimental results

The experimental concentration-response curves obtained were compared with the classical models of CA and IA. Some of the results are depicted in Figure 2, with the confidence intervals also reported for the experimental concentrationresponses curves. A more complete picture of the results can

Table 1. Inhibitory concentration (IC10 and IC50; 15 -min acute Microtox test; $\mathrm{mg} \mathrm{L}^{-1}$ ) values with the corresponding $95 \%$ confidence intervals (in parentheses) for the 10 tested binary combinations of pharmaceutical active compounds

\begin{tabular}{|c|c|c|c|c|c|c|}
\hline & & CTC & DCF & ASA & SMT & AMX \\
\hline CTC & IC10 & - & & & & \\
\hline \multirow[t]{2}{*}{ DCF } & IC10 & $0.68(0.41-0.96)$ & - & & & \\
\hline & IC50 & $10.1(8.72-11.5)$ & - & & & \\
\hline \multirow[t]{2}{*}{ ASA } & $\mathrm{IC} 10$ & $56.0(50.3-61.8)$ & $18.2(15.6-20.8)$ & - & & \\
\hline & IC50 & 378.6 (340.7-406.4) & $111.6(105.9-117.2)$ & - & & \\
\hline \multirow[t]{2}{*}{ AMX } & IC10 & $32.7(20.9-45.2)$ & $80.7(63.3-98.0)$ & $308.7(187.5-430.0)$ & $187.4(150.5-224.3)$ & - \\
\hline & IC50 & $3270.4^{\mathrm{a}}(2471.9-4068.9)$ & $846.2(783.8-908.6)$ & $3549.2^{\mathrm{a}}(2397.0-4501.4)$ & $2738.2^{\mathrm{a}}(2352.2-3135.2)$ & - \\
\hline
\end{tabular}

${ }^{\mathrm{a}}$ Values estimated with the Weibull model.

$\mathrm{AMX}=$ amoxicillin; $\mathrm{ASA}=$ acetylsalycilic acid $\mathrm{CTC}=$ chlortetracycline $\mathrm{DCF}=$ diclofenac sodium; $\mathrm{SMT}=$ sulfamethizole. 

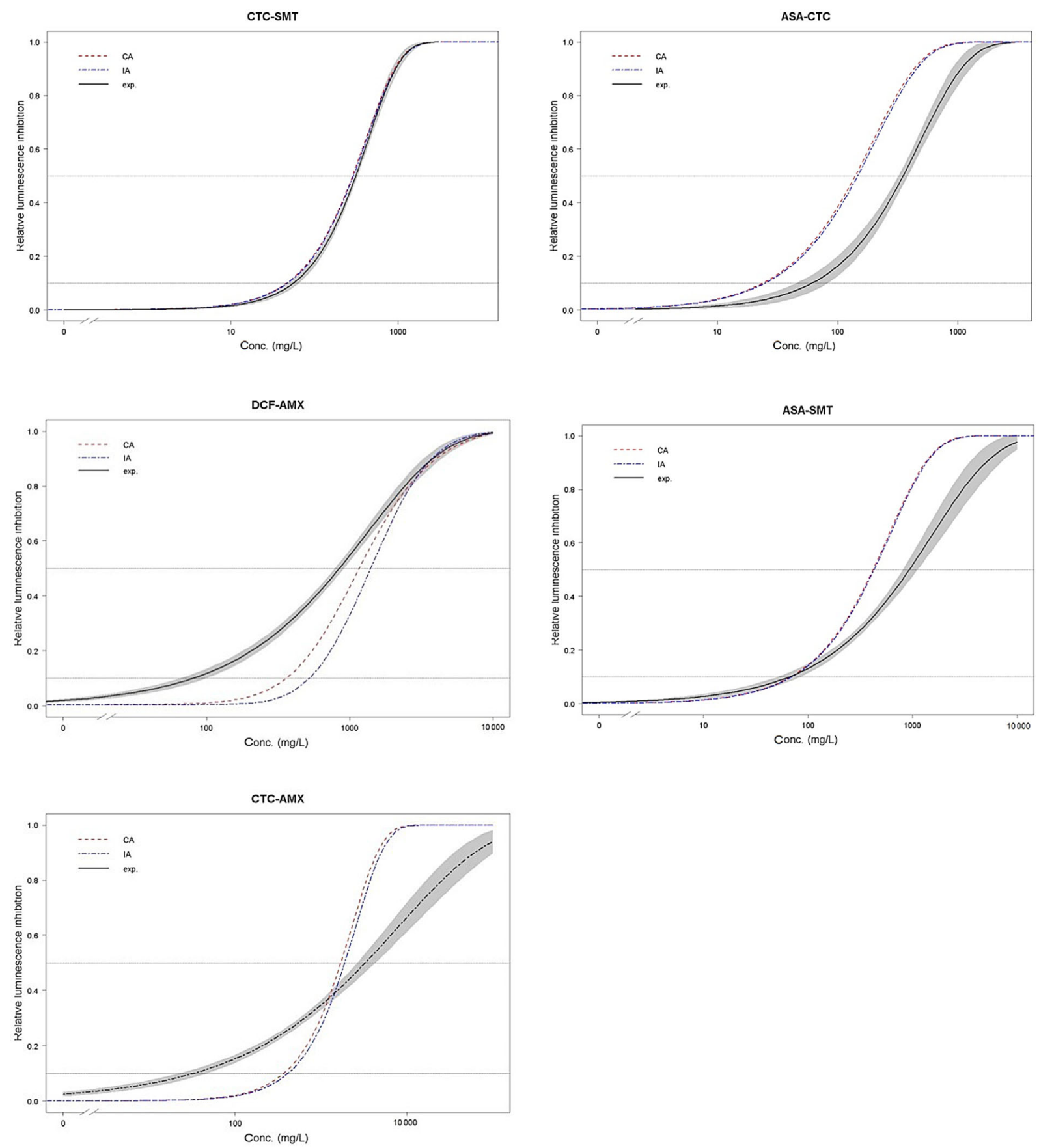

Figure 2. Predicted (concentration addition $=$ red line; independent action $=$ blue line) and observed toxicity values (black line) of binary mixtures (at inhibition concentration [IC10] concentration ratio) of veterinary pharmaceutical active compounds (gray shading $=95 \%$ confidence intervals). AMX $=$ amoxicillin; $\mathrm{ASA}=$ acetylsalycilic acid; $\mathrm{CTC}=$ chlortetracycline; $\mathrm{DCF}=$ diclofenac sodium; $\mathrm{SMT}=$ sulfamethizole.

be found in the Supplemental Data, Figure S1 and Table S3 (IC50 and IC10 predicted by CA and IA). The observed concentration responses are not always sufficiently described by the CA and IA models. In fact, with the exception of 3 binary combinations (chlortetracycline-sulfamethizole, diclofenac sodium-sulfamethizole, and acetylsalycilic aciddiclofenac sodium) and of the multicomponent mixture, the experimental toxicity data deviate from the conceptual expectations of the CA and IA. In 1 case (the binary mixture acetylsalycilic acid-chlortetracycline), both models clearly overestimate the bioluminescence inhibition response at all concentrations tested. In all the other cases, the extent of the deviation is mainly dependent on the mixture components and the effect level considered. In particular, for binary combinations showing greater observed toxicities than those predicted by models (e.g., chlortetracycline-diclofenac sodium, diclofenac sodium-amoxicillin), the deviations are considerably higher at lower concentrations. Recently, Marx et al. [33], using literature data, analyzed the presence of synergistic and antagonistic effects in antibiotic mixtures and found a potential increase in synergistic effects with decreasing concentrations. However, the authors concluded that because of the low number of investigations into the concentration dependency of mixture interactions and because 
of the dependency of this influence on the targeted organism and on the specific combination of chemicals, no general statement can be made at present.

In contrast, for acetylsalycilic acid-sulfamethizole (Figure 2) and acetylsalycilic acid-amoxicillin (Supplemental Data, Figure S1) combinations, the magnitude of deviation increased with an increase in concentration. However, in both cases, the deviations could be an artifact resulting from the lack of experimental data. (As reported above in the section Binary mixtures of pharmaceutical active compounds, the maximum percentage of inhibition reached was near 50\%.) Finally, both models seem to underestimate the effects of chlortetracyclineamoxicillin (Figure 2) and sulfamethizole-amoxicillin (Supplemental Data, Figure S1) mixtures at lower tested concentrations.

\section{Toxicological interactions of the tested pharmaceutical active} compounds in mixtures

The results reported in the section Predicted mixture toxicity by $C A$ and IA models and comparison with experimental results suggested possible interactions among the mixture components. The presence of synergism or antagonism in the mixtures of pharmaceutical active compounds (particularly in binary mixtures) or other pollutants has been reported in previous studies [14,21,23,34-37] and in the recent review by Backhaus [9]. Cleuvers [14] tested combinations of various pharmaceutical active compounds on algae and Daphnia and demonstrated that the evaluated compounds behaved as nonpolar narcotic compounds toward both organisms. In the algal test, the combination effect of a binary mixture of ibuprofen and diclofenac (2 nonsteroidal anti-inflammatory drugs [NSAIDs]) was well predicted by the CA model (as expected for substances with similar modes of action). However, in the Daphnia test, the effect of the mixture was stronger than that predicted by the CA model. In a further study, Cleuvers [34] evaluated a 4-component mixture of NSAIDs and confirmed that, in the algal test, the effect of increasing the number of NSAID components in the mixture was well predicted by the CA. In the Daphnia test, the results were quite different: at low doses (up to EC20/4), the mixture showed practically no effect (much lower than that predicted by the CA model), whereas at the higher concentrations, the effect was much higher than that predicted by the model. Rodea-Palomares et al. [21] found contrasting results when assessing the toxicological interactions of binary combinations of fibrates in V. fischeri and Anabaena. In fact, these authors reported that in the Vibrio test, the binary mixtures showed antagonism at low effect levels that turned into an additive effect or synergism at higher effect levels. However, in the Anabaena test, they found a strong synergism at the lowest effect levels that turned into a very strong antagonism at high effect levels. Gonzalez-Pleiter et al. [36] studied the toxicity of binary and multicomponent mixtures of 5 antibiotics to the green alga Pseudokirchneriella subcapitata and the cyanobacterium Anabaena CPB4337. The study found a clear predominance of synergistic behavior for most of the antibiotic interactions in these organisms. In particular, the amoxicillin-tetracycline combination tested on cyanobacterium was strongly synergistic. Conversely, the same authors [36] found a dual behavior with rapid changes of interactions (from strong synergism to strong antagonism) when tetracycline in combination with erythromycin was tested in Anabaena. Brezovsek et al. [37] tested the toxicity of 3 binary mixtures of antineoplastic drugs to the green alga $P$. subcapitata and the cyanobacterium Synechococcus leopolinensis and compared their results with predictive models (CA and IA).
The binary combination of 5-fluorouracil and imatinib had a synergistic effect for $P$. subcapitata and an antagonistic effect on S. leopolinensis. Based on these results, the authors noted the importance of species-dependent interactions between the pharmaceuticals in a mixture.

Backhaus [9] highlighted that, for pharmaceuticals, the synergisms and antagonisms were specific for the tested mixture and the toxicity test. In particular, synergistic mixtures seemed to be largely confined to mixtures of only a few compounds, usually not more than 2 or 3 . In contrast, synergistic or antagonistic mixture effects were rarely observed in toxicity tests for multicomponent mixtures. Backhaus [9] argued that this phenomenon might be explained by the presence of a sort of buffering against synergistic or antagonistic interactions when the contributions of the latter are not predominant.

Warne and Hawker [38], in their work on the funnel hypothesis, explained the variation in the toxicity of multicomponent mixtures of nonspecific toxicants (narcotics) and concluded that mixtures of this type of toxicants tended to approach additivity (becoming less synergistic or antagonistic) as the number of components increased.

In the present study, to evaluate the presence and eventually quantify the nature of such interactions (e.g., synergism, antagonism) at any effect level, the combination index method was applied. Figure 3 shows a plot of the combination index values obtained as a function of effect levels $\left(f_{a}\right)$ for all of the tested mixtures. The corresponding combination index values at the main representative $f_{a}$ levels (effect level of fraction inhibited with respect to the control) are reported in the Supplemental Data, Table S3. The $f_{a}$-combination index plots allowed us to observe, at any $f_{a}$ level, the trend of the nature of the interactions among pharmaceutical active compounds in mixtures. In Figure 3, combination index values up to the effect level $\left(f_{a}\right)$ of 0.5 are reported, for those binary mixtures for which the entire concentration-response curves were not obtained.

From the analysis of Figure 3 and Supplemental Data, Table $\mathrm{S} 4$, the following inferences may be made. First, for almost all the tested mixtures, $f_{a}$-combination index plots and combination index values seems to confirm the presence of interactions among the components. The only exceptions are the chlortetracyclinesulfamethizole, diclofenac sodium-sulfamethizole, and acetylsalycilic acid-diclofenac sodium binary combinations, which are fairly additive (combination index $\sim 1$ ) at all the effect levels considered. Second, synergism or antagonism seem to be independent of the mode of action toward the tested organism. As highlighted in our previous study [24], we demonstrated that, with the exception of chlortetracycline, all of the selected pharmaceutical active compounds act as polar narcotic compounds to $A$. fischeri. In agreement with the previously reported results of Cleuvers [14] on Daphnia, even if these substances share (broadly speaking) the same mechanism of action, we found that the effect of the binary mixtures deviates from CA predictions. In addition, binary combinations composed of substances with different mechanisms of action (i.e., those including chlortetracycline) show very different and unpredictable behaviors. Third, clear synergistic effects are present for the chlortetracycline-diclofenac sodium and diclofenac sodiumamoxicillin combinations up to the effect level $\left(f_{a}\right)$ of 0.65 to 0.70 . The results obtained for chlortetracycline-diclofenac sodium seem to be in line with those of previous studies. Olajuyigbe and Afolayan [39] tested combinations of tetracycline and amoxicillin in 8 different kinds of antibiotic-resistant bacteria (either gram-negative or gram-positive bacteria) and found that the combinations often had a synergistic effect. Interestingly the 


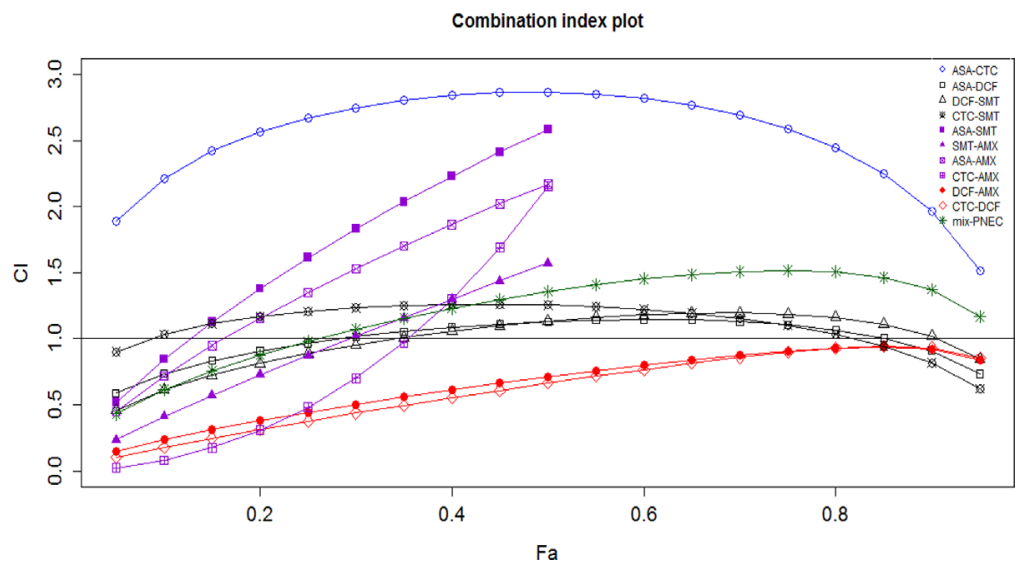

Figure 3. Combination index plot $\left(f_{a}\right.$-combination index plot) for binary and multicomponent mixtures of tested pharmaceutical active compounds toward Aliivibrio fischeri. The combination index values are plotted as a function of the fractional inhibition of bioluminescence $\left(f_{a}\right)$ by computer simulation (CompuSyn). Combination index $<1,=1$, and $>1$ indicate synergism, the additive effect, and antagonism, respectively [32]. AMX $=$ amoxicillin; ASA $=$ acetylsalycilic acid; $\mathrm{CTC}=$ chlortetracycline; $\mathrm{DCF}=$ diclofenac sodium; $\mathrm{SMT}=$ sulfamethizole.

same authors [39] also found that the synergistic effects were concentration dependent between the 2 antibiotics. In contrast, acetylsalycilic acid-chlortetracycline clearly interacted in an antagonistic way over the whole range of effect levels. Fourth, for other binary combinations (chlortetracycline-amoxicillin, sulfamethizole-amoxicillin acetylsalycilic acid-sulfamethizole, acetylsalycilic acid-amoxicillin), a somewhat heterogeneous pattern is observed, with interactions changing from synergism at low $f_{a}(0.1-0.3)$ to a clear antagonism within a narrow range of concentrations. The presence of synergism at lower concentrations and the tendency toward additivity, or antagonism, at the higher levels is not easy to explain, because the nature of the interactions are complex and mostly unknown. In any case, as discussed previously, similar results are found in the literature [21,35,36]. For instance, Magdaleno et al. [40] tested the effects on the growth of $P$. subcapitata of combinations of several antibiotics and found that at low concentrations $\left(<10 \mathrm{mg} \mathrm{L}^{-1}\right)$, all binary combinations showed synergism, whereas in almost all cases, at the higher concentrations tested, the response became close to the predictions.

In some cases, the nature of the interactions seems to be related to the presence of certain compounds. For instance, at the lower tested concentrations, the presence of the anti-inflammatory diclofenac sodium seems to enhance the effects of the antibiotics amoxicillin, chlortetracycline, and (to a lesser extent) sulfamethizole). Several studies have noted that the efficiency of some antibiotics (streptomycin, gentamicin, tetracycline, and ampicillin) toward bacteria can be synergistically enhanced by the presence of diclofenac sodium [41-45]. These studies found that diclofenac sodium has the pability to damage the bacterial membrane [46], and to favor alteration in the permeability of the microorganism to antibiotics $[45,47,48]$. In contrast, in the present study, the presence of the anti-inflammatory acetylsalycilic acid seems to weaken their toxicity.

Finally, the multicomponent mixture showed no strong synergism or antagonism. In fact, the combination index values ranged from 0.61 (IC10) to 1.37 (IC90) until the tested effect level. The results we obtained in the toxicity test for the multicomponent mixtures seem to support the buffering hypothesis proposed by Backaus [9].

As reported by Kortenkamp et al. [49], synergistic cases are highly specific for specific compounds, their concentrations, and their mixture ratios. In the present study, the concentrations of the tested mixtures giving synergistic responses are above the environmentally relevant concentrations and in addition, for our experimental purpose, substances were combined in equal ratios according to their individual IC10 value, which could be quite different from the real ratio at which toxicants might be found in the environment. Thus responses from naturally occurring combinations could be different.

\section{CONCLUSIONS}

The toxicity of binary and multicomponent mixtures of widely used veterinary pharmaceuticals was evaluated using the Microtox test system. For the multicomponent mixtures tested, the results indicated that at concentrations corresponding to the sum of individual PNECs, no effects on A. fischeri were present. This finding suggested that PNEC values were sufficiently protective when the investigated pharmaceutical active compounds were contemporaneously present in aquatic environments, at least for microorganisms and for short-term exposure. By comparing the experimental toxicity curves with those predicted by the CA and IA models, it was possible to hypothesize the presence, in binary mixtures, of toxicological interactions between the components, which, in several cases, leads to synergistic effects at low concentrations (particularly when either diclofenac sodium or amoxicillin was 1 of the components). The subsequent application of the combination index method confirmed the presence of synergistic and antagonistic interactions. Finally, synergism or antagonism seemed to be independent of the mode of action of the toxicants tested. This seems to confirm that for combinations of pharmaceutical active compounds, use of the predictive models may have some limitations in terms of assessment of the real mixture toxicity.

Supplemental Data-The Supplemental Data are available on the Wiley Online Library at DOI: 10.1002/etc.3686.

Data Availability - Data, associated metadata, and calculation tools are available from the corresponding author (v.dinica@campus.unimib.it).

\section{REFERENCES}

1. Battaglin WA, Fairchild JF. 2002. Potential toxicity of pesticides measured in midwestern streams to aquatic organisms. Water Sci Technol 45:95-102. 
2. Monteiro SC, Boxall ABA. 2010. Occurrence and fate of human pharmaceuticals in the environment. Rev Environ Contam T 202: 53-154.

3. Schäfer RB, von der Ohe PC, Kühne R, Schüürmann G, Liess M, 2011. Occurrence and toxicity of 331 organic pollutants in large rivers of north Germany over a decade (1994 to 2004). Environ Sci Technol 45:6167-6174.

4. Malaj E, von der Ohe P, Grote M, Kühne R, Mondy CP, UsseglioPolatera P, Brack W, Schäfer RB. 2014. Organic chemicals jeopardize the health of freshwater ecosystems on the continental scale. Proc Natl Acad Sci U S A 111:9549-9554.

5. European Commission. 2009. Combination effects of chemicalsCouncil conclusions 17820/09. Adopted by the Council on 22 December 2009. [cited 2016 January 28]. Available from: http:// register.consilium.europa.eu/doc/srv?l=EN\&f=ST\%2017820\%20200 $9 \% 20$ INIT

6. European Commission. 2012. The combination effects of chemicalsChemical mixtures. Communication from the Commission to the Council. COM, p. 252 (final). [cited 2016 January 28]. Available from: http://eurlex.europa.eu/legal-content/EN/TXT/PDF/?uri=CELEX:52012DC0252\& from $=\mathrm{EN}$

7. Barata C, Baird DJ, Nogueira AJA, Soares A, Riva MC. 2006. Toxicity of binary mixtures of metals and pyrethroid insecticides to Daphnia magna Straus. Implications for multi-substance risks assessment. Aquat Toxicol 78:1-14.

8. Vighi M, Villa S. 2013. Ecotoxicology: The challenges for the 21st century: Review. Toxics 1:18-35.

9. Backhaus T. 2014. Medicines, shaken and stirred: A critical review on the ecotoxicology of pharmaceutical mixtures. Philos Trans $R$ Soc Lond B Biol Sci 369:20130585.

10. Loewe S, Muischnek H. 1926. Effect of combinations: Mathematical basis of problem. N-S Arch Exp Pathol Physiol 114:313-326.

11. Bliss CI. 1939. The toxicity of poisons applied jointly. Ann Appl Biol 26:585-615.

12. Backhaus T, Altenburger R, Boedeker W, Faust M, Scholze M, Grimme LH. 2000. Predictability of the toxicity of a multiple mixture of dissimilarly acting chemicals to Vibrio fischeri. Environ Toxicol Chem 19:2348-2356.

13. Backhaus T, Scholze M, Grimme LH. 2000. The single substance and mixture toxicity of quinolones to the bioluminescent bacterium Vibrio fischeri. Aquat Toxicol 49:49-61.

14. Cleuvers M. 2003. Aquatic ecotoxicity of pharmaceuticals including the assessment of combination effects. Toxicol Lett 142:185-194.

15. Faust M, Altenburger R, Backhaus $T$, Blanck $H$, Boedeker W, Gramatica P. 2003. Joint algal toxicity of 16 dissimilarly acting chemicals is predictable by the concept of independent action. Aquat Toxicol 63:43-63.

16. Backhaus T, Faust M. 2012. Predictive environmental risk assessment of chemical mixtures: A conceptual framework. Environ Sci Technol 46:2564-2573.

17. Backhaus T, Faust M, Scholze M, Gramatica P, Vighi M, Grimme LH. 2004. Joint algal toxicity of phenylurea herbicides is equally predictable by concentration addition and independent action. Environ Toxicol Chem 23:258-264.

18. Villa S, Migliorati S, Monti GS, Vighi M. 2012. Toxicity on the luminescent bacterium Vibrio fischeri (Beijerinck). II: Response to complex mixtures of heterogeneous chemicals at low levels of individual components. Ecotoxicol Environ Saf 86:93-100.

19. Chou T-C, Talalay P. 1983. Analysis of combined drug effects: A new look at a very old problem. Trends Pharmacol Sci 4:450-454.

20. Chou T-C. 2006. Theoretical basis, experimental design, and computerized simulation of synergism and antagonism in drug combination studies. Pharmacol Rev 58:621-681.

21. Rodea-Palomares I, Petre AL, Boltes K, Leganés F, Perdigón-Melón JA, Rosal R, Fernández-Piñas F. 2010. Application of the combination index (CI)-isobologram equation to study the toxicological interactions of lipid regulators in two aquatic bioluminescent organisms. Water Res 44:427-438.

22. Boltes K, Rosal R, García-Calvo E, 2012. Toxicity of mixtures of perfluorooctane sulphonic acid with chlorinated chemicals and lipid regulators. Chemosphere 86:24-29.

23. Rodea-Palomares I, Leganés F, Rosal R, Fernández-Piñas F. 2012. Toxicological interactions of perfluorooctane sulfonic acid (PFOS) and perfluorooctanoic acid (PFOA) with selected pollutants. J Hazard Mater 201-202:209-218.

24. Di Nica V, Villa S, Finizio A. 2016. Toxicity of individual pharmaceuticals and their mixtures to Aliivibrio fischeri: Experimental results for single compounds and considerations of their mechanisms of action and potential acute effects on aquatic organisms. Environ Toxicol Chem 36:807-814 (this issue).

25. Vighi M, Migliorati S, Monti GS. 2009. Toxicity on the luminescent bacterium Vibrio fischeri (Beijerinck). I: QSAR equation for narcotics and polar narcotics. Ecotoxicol Environ Saf 72:154-161.

26. European Medicines Agency. 2006. Guideline on the environmental risk assessment of medicinal products for human use. 01 June 2006 Doc. Ref. EMEA/CHMP/SWP/4447/00 corr 1. London, UK.

27. Finizio A, Vighi M. 2014. Predicted no effect concentration (PNEC). In Wexler P, ed, Encyclopedia of Toxicology, 3rd ed, Vol 3. Elsevier/ Academic Press, New York, NY, USA, pp 1061-1065.

28. R Core Team. 2014. R: A Language and Environment for Statistical Computing. Vienna, Austria. [cited 2015 December 13]. Available from: http://www.R-project.org/.

29. Ritz C, Streibig JC. 2005. Bioassay analysis using R. J Stat Softw 12: Issue 5.

30. Chou T-C. 1976. Derivation and properties of Michaelis-Menten type and Hill type equations for reference ligands. J Theor Biol 59:253-254, IN1,255-276.

31. Chou T-C, Talalay P. 1984. Quantitative analysis of dose-effect relationships: Rhe combined effects of multiple drugs or enzyme inhibitors. Adv Enzyme Regul 22:27-55.

32. Chou TC, Martin N, 2005. CompuSyn for Drug Combinations: PC Software and User's Guide: A Computer Program for Quantification of Synergism and Antagonism in Drug Combinations and the Determination of IC50 and ED50 and LD50 Values. ComboSyn, Paramus, NJ, USA.

33. Marx C, Mühlbauer V, Krebs P, Kuehn V. 2015. Environmental risk assessment of antibiotics including synergistic and antagonistic combination effects. Sci Total Environ 524-525:269-279.

34. Cleuvers M. 2004. Mixture toxicity of the anti-inflammatory drugs diclofenac, ibuprofen, naproxen, and acetylsalicylic acid. Ecotoxicol Environ Saf 59:309-315.

35. Rosal R, Rodea-Palomares I, Boltes K, Fernández-Piñas F, Leganés F, Petre A. 2010. Ecotoxicological assessment of surfactants in the aquatic environment: Combined toxicity of docusate sodium with chlorinated pollutants. Chemosphere 81:288-293.

36. Gonzalez-Pleiter M, Gonzalo S, Rodea-Palomares I, Leganes F, Roberto Rosal R, Karina Boltes K, Marco E, Fernandez-Pinas F. 2013. Toxicity of five antibiotics and their mixtures towards photosynthetic aquatic organisms: Implications for environmental risk assessment. Water Res 47:2050-2064.

37. Brezovsek P, Elersek T, Filipic M. 2014 Toxicities of four antineoplastic drugs and their binary mixtures tested on the green alga Pseudokirchneriella subcapitata and the cyanobacterium Synechococcus leopoliensis. Water Res 52:168-177.

38. Warne MStJ, Hawker DW. 1995. The number of components in a mixture determines whether synergistic and antagonistic or additive toxicity predominate: The funnel hypothesis. Ecotoxicol Environ Saf 31:23-28.

39. Olajuyigbe OO, Afolayan AJ. 2012. Synergistic interactions of methanolic extract of Acacia mearnsii de wild. with antibiotics against bacteria of clinical relevance. Int J Mol Sci 13:8915-8932.

40. Magdaleno A, Saenz ME, Juárez AB, Moretton J. 2015. Effects of six antibiotics and their binary mixtures on growth of Pseudokirchneriella subcapitata. Ecotoxicol Environ Saf 113:72-78.

41. Chattopadhyay D, Dastidar SG, Chakrabarty AN. 1987. Determination of in vitro activity of methdilazine, an antihistamine, and its synergism with aminoglycoside antibiotics. Indian J Med Microbiol 5:171-177.

42. Ray S, Chattopadhyay D, Dastidar SG, Chakrabarty AN. 1990. Studies on synergism between penicillins and ambodryl (bromodiphenhydramine $\mathrm{HCl}$ ), an antihistamine with antimicrobial property. Indian J Exp Biol 28:253-258.

43. Dastidar SG, Chakraborty $\mathrm{P}$, Mookerjee M, Ganguly M, Chakrabarty AN. 1994. Studies on the existence of synergism between different antibiotics and a phenothiazine tranquilizer, promazine, possessing antimicrobial property. Acta Microbiol Immunol Hung 41:41-49.

44. Dutta NK, Mazumdar K, Dastidar SG, Park JH. 2007. Activity of diclofenac used alone and in combination with streptomycin against Mycobacterium tuberculosis in mice. Int J Antimicrob Ag 30: 336-340.

45. Dutta NK, Annadurai S, Mazumdar K, Dastidar SG, Kristiansen JE, Molnár J, Martins M, Amaral L. 2007. Potential management of resistant microbial infections with a novel non-antibiotic: The antiinflammatory drug diclofenac sodium. Int J Antimicrob Ag 30: 242-249. 
46. Dutta NK, Mazumdar K, Baek MW, Kim DJ, Na YR, Park SH, Lee HK, Lee BH, Park JH. 2008. In vitro efficacy of diclofenac against Listeria monocytogenes. Eur J Clin Microbiol Infect Dis 27:315-319.

47. Amaral L, Lorian V. 1991. Effects of chlorpromazine on the cell envelope proteins of Escherichia coli. Antimicrob Agents Chemother 35:1923-1924.
48. Viveiros M, Amaral L. 2001. Enhancement of antibiotic activity against poly-drug resistant Mycobacterium tuberculosis by phenothiazines. Int $J$ Antimicrob Ag 17:225-228.

49. Kortenkamp A, Backhaus T, Faust M. State of the art report on mixture toxicity. 2009. [cited 2016 January 28]. Available from: http://ec.europa. eu/environment/chemicals/pdf/report_Mixture\%20toxicity.pdf 